\section{Scaling Laws in Mechanics of Failure}

\section{By Zdeněk P. Bažant,' Fellow, ASCE}

ABstract: Scaling laws are the most fundamental aspect of every physical theory Recently, the problem of scaling law and size effect in the theories of structura ailure has received considerable attention, particularly with regard to distributed damage and nonlinear fracture behavior. The paper presents a rigorous natheFirst it is shown that the scaling law is a power law if and only if a characteristic dimension is absent. For all the theories in which the faiture condition is expressed in terms of stress or strain only, including elasticity with a strength limit plasticity. and continuum damage mechanics, the nominal strength of the structure is shown to be independent of its size. For linear-clastic fracture mechanics, in which the foilure criterion is expressed in terms of encrgy per unit area the scaling law for the nominal strength is shown to be (size $)^{-1 / 2}$. provided that the cracks in structurcs of different sizes are gcometrically similar. When the failure condition involves both the stress (or strain) and the encrgy per unit area, which is typical of quasibritte materials, the scaling law represents a gradual transition from the strength theory, which is asymptotically approached for very small sizes, to LEFM, which is asymptotically approached for very large sizes. The size effect described by Weibul totically approached for very large sizes. The size effect described by Weibul for its inapplicability to quasibrittle materiats are explained. Finally, floating clastic plates with latge bending fractures and a negligible process-zone size are shown to pxhibit an anomalous scaling law, such that the nominal strength is proportional to (size) 3.78 . and another anomalous size effect of the type (size)-2/5, pertinent to one recent theory of borehole breakout, is pointed out

\section{INTRODUCTION}

Until about a decade ago, it was generally believed that the size effect in structural failure is of statistical origin, caused by randomness of material strength. Accordingly, it was thought that studies of the size effect should be left to statisticians, and that the size effect should be relegated to the safety factor.

Recently, however, it has been discovered that an important and often dominant size effect can be of purely mechanical and deterministic origin. Such a size effect can be caused by the influence of the release of the stored elastic energy on the nominal strength of the structure Many studies, both experimental and theoretical, have already dealt with this type of size effect, and an approximate size-effect law applicable to structures in which fracture is preceded by distributed cracking in a large fracture-process zone has been formulated (Bažant 1983, 1984; Bažant and Pfeiffer 1987; Bažant and Kazemi 1990)

The problem of size effect is particularly important to structural and geotechnical engineers, and even more to geophysicists. To mechanical and aerospace engineers, the problem is practically of lesser interest, because most structures are tested at full size, and the main problem is extrapolation in time rather than size (i.e., the prediction of the structure life). Civil engineers, however, must inevitably extrapolate from reduced-scale labo-

'Walter P. Murphy, Prof. of Civ. Engrg., Northwestern Univ., Evanston, IL 60201

Note. Discussion open until February 1, 1994. To extend the closing date one month, a written request must be filed with the ASCE Manager of Journals. The manuscript for this paper was submitted for review and possible publication on October 6, 1992. This paper is part of the Journal of Engineering Mechamics, Vol $119, \mathrm{~N}, 9 . \mathrm{September,} \mathrm{1993.} \mathrm{OASCE,} \mathrm{ISSN} \mathrm{0733-9399/93/0009-1828/ \$ 1.00} \mathrm{+}$ per page. Paper No. 4898 ratory tests to real structures, which are too large to be tested systematically. Realization of the problems involved in this extrapolation has recently led to a surge of interest in this subject in civil engineering

The problem of size effect, however, is important in all fields. If the scaling law for some physical phenomenon is not understood, the phenomenon itself is not understood. Indeed, the discovery that the classical Newtonian mechanics fails at very large dimensions led to the theory of relativity, and the discovery that it fails at very small dimensions led to quantum mechanics. Likewise, the problem of scaling played a central role in fluid mechanics; recall for example, the boundary-layer theory for flow in thin surface layers, Reynolds number, and so forth. Simply, the problem of scaling is the most fundamental aspect of any physical theory and ought to be carefully studied, not only by civil engineers but also by mechanical and aerospace engineers.

The purpose of this paper is to present a rigorous and detailed derivation of the basic scaling laws of structural mechanics, with a focus on the deterministic type of size effect [a detailed study of the statistical size effect has been presented recently by Bažant and Xi (1991a, b)]. Various types of power-scaling laws will be examined in relation to the type of failure criterion, and the distinctions between material-strength theories and fracture mechanics will be clarified. Some surprising anomalous kinds of size effect encountered in plate bending will be also discussed and explained. The present investigation is strictly theoretical and attempts to synthesize the experience from numerous recent experimental and numerical studies [for a review, see Bažant and Kazemi (1990)].

\section{NOMINAL STRENGTH AND DEFINITION OF SIZE EFFECT}

The size effect is defined by comparing geometrically similar structures of different sizes (in the case of notched or fractured structures, the geometric similarity means that the notches or initial cracks are also geometrically similar). We denote as $Y$ the response quantity whose size dependence is to be determined-for example, the nominal strength, the maximum deflection, or the maximum strain. In this paper, our interest is in comparing the nominal strength (or nominal stress at failure) $Y=\sigma_{N}$, which is defined as

$\sigma_{N}=c_{N} \frac{P_{u}}{b D} \quad($ for $2 D)$

or

$\sigma_{N}=c_{N} \frac{P_{u}}{D^{2}}$

(for $3 D$ )

in which $P_{u}=$ maximum (ultimate) load; $b=$ structure thickness in the case of two-dimensional similarity; $D=$ characteristic dimension (or characteristic size), which can be chosen arbitrarily (for instance, as the depth of beam, the span, the half span, the notch depth, and so forth); and $c_{N}=$ coefficient introduced for convenience if one desires $\sigma_{N}$ to correspond to some commonly used stress formulas. For example, in the case of bending of a simply supported beam of span $L$ and depth $h$, with a concentrated load $P$ at midspan, one may set $D=h$ and introduce $\sigma_{N}$ as the maximum elastic bending stress; that is, $\sigma_{N}=3 P_{u} L / 2 b D^{2}$ in which case one has (1) 
with $c_{N}=1.5 \mathrm{~L} / D=$ constant. But one can equally well take the characteristic dimension as $D=L=$ span, and denoting $h=$ beam depth, one has (1) where now $c_{N}=3 L^{2} / 2 h^{2}=$ constant. Or, one can use for $\sigma_{N}$ the formula for maximum plastic bending stress $\sigma_{N}=P L / b D^{2}$ where $D=$ beam depth, which may be written as (1) with $c_{N}=L / D=$ constant. Upon setting $D=L$, one may write this as (1) with $D=L$, in which case $c_{N}=L^{2} / h^{2}$ $=$ constant. One can also choose $\sigma_{N}=P_{u} / b h=$ average shear stress, and then, setting $D=h$, one has $c_{N}=1$, while setting $D=L$, one has $c_{N}=$ $L / h=$ constant

\section{GENERAL SCALING LAW IN ABSENCE OF}

\section{CHARACTERISTIC LENGTH}

Let us first consider those theories in which there is no characteristic length. This means that the scaling ratio $Y / Y$ of the corresponding responses $Y$ and $Y$ depends only on the size ratio $\lambda=\bar{D} / D$ of two different sizes $D$ and $D$ but is independent of the choice of the reference size $D$. Plasticity, elasticity with a strength limit, continuum damage mechanics (without nonlocal concepts), and also linear-elastic fracture mechanics (LEFM) belong to this class of theories, as do many other theories in physics. As is well known, the scaling law for all these theories is a power law. We will now show it by adapting an argument used in fluid mechanics (Barenblatt 1979 1987). Let the scaling law be $f(\lambda)$, that is

$\frac{\bar{Y}}{Y}=f(\lambda)$

where $f=$ unknown function that we want to find. Considering another structure size $D=\mu D$ with the corresponding response $Y$, we have

$\frac{\bar{Y}}{Y}=f(\mu)$

Now, because there exists no characteristic size, the size $\bar{D}$ can alternatively be chosen as the reference size. In that case, (2) implies that

$\frac{\tilde{Y}}{\bar{Y}}=f\left(\frac{\mu}{\lambda}\right)$

Substituting now the ratio of (2) and (3) into (4), we obtain

$f\left(\frac{\mu}{\lambda}\right)=\frac{f(\mu)}{f(\lambda)}$

This is a functional equation from which the function $f(\lambda)$ can be solved To this end, we differentiate (5) with respect to $\mu$ and then set $\mu=\lambda$

$\frac{f^{\prime}(1)}{\lambda}=\frac{f^{\prime}(\lambda)}{f(\lambda)}$

in which $f^{\prime}=$ derivative of function $f$. The last equation is a differential equation for the unknown function $f$, which can be easily solved by separation of variables. With the notation $f^{\prime}(1)=m=$ constant, the integral is $\ln f(\lambda)=m \ln \lambda+C$, and determining the integration constant $C$ from the condition $C=\ln f(1)=0$ for $\lambda=1$, we have $f(1)=1$. So we finally conclude that function $f$ must be a power function

$f(\lambda)=\lambda^{\prime \prime}$

The power-scaling law we obtained must hold for every physical system in which there is no characteristic dimension. This includes plasticity or elasticity with a strength limit. Further, this includes LEFM. This is so despite the fact that the tensile strength $f_{1}^{\prime}$, Young's elastic modulus $E$, and fracture energy $G_{f}$ can be combined to give a length quantity $l_{0}=E G_{f} / f^{\prime 2}$ (which has often been called the characteristic length, but is better called the characteristic fracture process-zone size because the former term means something else in the previously established terminology of nonlocal continuum theory). The reason that the presence of $l_{0}$ in LEFM does not destroy the validity of the power-law scaling (as will also be shown by another approach later) is that, in LEFM, the fracture-process zone is treated as a point, and that there is no change in failure mechanism associated with $l_{1}$ (this is in contrast to nonlinear fracture mechanics, c.g., the crack-band model or the cohesive-crack model).

To prove the converse, i.e., that there is no characteristic size if the scaling law is a power law, is almost trivial. It is given that $f(\lambda)=\lambda^{m}$, which means that $\bar{Y} / Y=(\bar{D} / D)^{m}$ and $\bar{Y} / Y=(\tilde{D} / D)^{\prime \prime}$. Taking the ratio of these two expressions, we then have $\bar{Y} / \bar{Y}=(\bar{D} / D)^{\prime \prime \prime} /(\bar{D} / D)^{\prime \prime}=(\bar{D} / \bar{D})^{m}$. So, when $\bar{D}$ is taken as the reference size, the scaling law has exactly the same form as when $D$ is taken as the reference size. Consequently, the scaling law for a certain theory is a power law if and only if (i.e., 'iff') no characteristic dimension is present in the theory.

For example, the Weibull-type statistical strength theory in which the spatial density of the material failure probability is given by a power law with a zero threshold leads to a power-type size effect. This implics that there is no characteristic length. It follows that this theory is unrealistic for structures where a characteristic length is obviously provided by the material inhomogeneities or the size of the fracture-process zone [this conclusion was reached in a different manner in Bažant and Xi (1991)].

\section{SCALING LAW FOR BOUNDARY VALUE PROBLEM OF}

\section{CONTINUUM MECHANICS}

Geometrically similar structures of different sizes are related by the affine transformation (affinity), which is the transformation of change of scale

$\bar{x}_{i}=\lambda x_{i}$

where $x_{i}=$ Cartesian coordinates for the reference structure of characteristic dimension (size) $D ; \bar{x}_{i}=$ coordinates for a geometrically similar scaled structure (Fig. 1); and $\lambda=\bar{D} / D$ where $\bar{D}$ is the characteristic dimension of the scaled structure. The overbars are used to label the quantities referring to the scaled structure. For the sake of brevity, we will denote $\partial / \partial x_{i}=\partial_{i}$, $\partial / \partial \bar{x}_{i}=\bar{\partial}_{i}$. From the chain rule of differentiation, $\partial_{i}=\lambda \bar{\partial}_{i}, \bar{\partial}_{i}=\lambda^{-1} \partial_{i}$

For the reference structure of size $D$ and the similar scaled structure of size $D$, the field equations and the boundary conditions are

$\partial_{i} \sigma_{i j}+f_{i}=0$
$\dot{\partial}_{j} \bar{\sigma}_{i j}+\bar{f}_{i}=0$




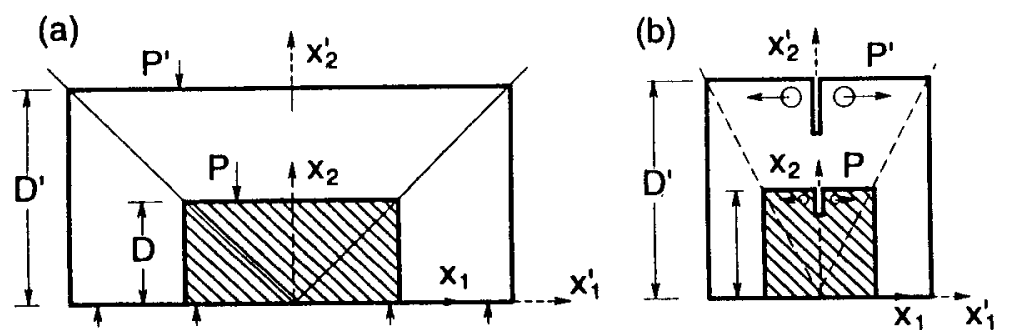

FIG. 1. Geometrically Similar Structures: (a) without Cracks; (b) with Similar Cracks

$\varepsilon_{i j}=\frac{\partial_{j} u_{i}+\partial_{i} u_{j}}{2}$ for $D$

$\bar{\varepsilon}_{i j}=\frac{\bar{\partial}_{j} \bar{u}_{i}+\bar{\partial}_{i} \bar{u}_{j}}{2} \quad$ for $\bar{D}$

$\sigma_{i j} n_{j}=p_{i} \quad$ on $\Gamma_{1} \quad$ for $D$

$\bar{\sigma}_{i j} \bar{n}_{j}=\bar{p}_{i} \quad$ on $\bar{\Gamma}_{1} \quad$ for $\bar{D}$

$u_{i}=U_{i} \quad$ on $\mathrm{r}_{2} \quad$ for $D$

$\bar{u}_{i}=\bar{U}_{i} \quad$ on $\bar{\Gamma}_{2} \quad$ for $\bar{D}$

in which $\sigma_{i j}$ and $\varepsilon_{i j}=$ stresses and strains in Cartesian coordinate $x_{i}$ (the strains are assumed to be small); $u_{i}=$ displacements of material points; $\Gamma_{1}$ and $\Gamma_{2}=$ the portions of the boundary with prescribed surface tractions $p$ and with prescribed displacements $U_{i} ; f_{i}=$ prescribed volume forces; and $n_{i}=\bar{n}_{i}=$ direction cosines of unit outward normals on the stress boundary.

From (7), we already know that the scaling law must be a power function Let us now assume that the displacements are related by the scaling law

$\bar{u}_{i}=\lambda^{m+1} u_{i}$

where $m=$ unknown exponent. Substituting this into the differential equations and boundary conditions (9)-(12), we find $\bar{\varepsilon}_{i j}=\lambda^{m}\left(\partial_{j} u_{i}+\partial_{i} u_{j}\right) / 2$. According to (10), the following transformation rules then ensue:

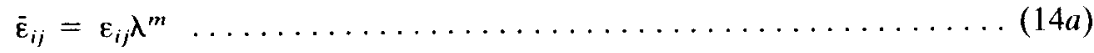

$\overline{\boldsymbol{\sigma}}_{i j}=\sigma_{i j} \lambda^{m}$

$\bar{\sigma}_{N}=\sigma_{N} \lambda^{m}$

$\bar{p}_{i}=p_{i} \lambda^{m}$

$\tilde{f}_{i}=f_{i} \lambda^{m-1}$

$\bar{u}_{i}=u_{i} \lambda^{m+1}$

These rules indicate how a solution for one size can be transformed to a solution for another size. However, the value of $m$ is indeterminate. To determine it, we cannot ignore the constitutive law and the failure condition. Next we consider in this regard two important special cases.

\section{ELASTIC-PLASTIC STRUCTURES}

The constitutive relation and the condition of no failure (either the yield condition or the condition of allowable stress) have the general form

$\sigma_{i j}=\mathscr{F}_{i j}\left(\varepsilon_{k m}\right)$

$\phi\left(\sigma_{i j}, \varepsilon_{i j}\right)<\sigma_{0}$

in which $\mathscr{F}_{i j}=$ tensor-valued functions or functionals of a tensorial argument; $\phi=$ nonlinear scalar function of tensorial arguments; and $\sigma_{0}=$ material yield limit or allowable stress limit. After transformation of scale, (16) takes the form $\bar{\sigma}_{i j}=\overline{\mathscr{F}}_{i j}\left(\bar{\varepsilon}_{k m}\right), \phi\left(\bar{\sigma}_{i j}, \bar{\varepsilon}_{i j}\right)<\sigma_{0}$. Since at least function $\phi$ (and possibly function $\mathscr{F}_{F}$ ) is nonlinear, this is possible if and only if $\bar{\sigma}_{i j}$ $=\sigma_{i j}$ and $\bar{\varepsilon}_{k m}=\varepsilon_{k m}$, which means that $m=0$. The transformation rules from (13) and (15) then become

$\bar{u}_{i}=\lambda u_{i}$

$\bar{\varepsilon}_{i j}=\varepsilon_{i j}$

$\bar{\sigma}_{i j}=\sigma_{i j}$

$\hat{p}_{i}=p_{i}$

$\vec{f}_{i}=f_{i} / \lambda$

$\bar{u}_{i}=u_{i} \lambda$

\section{Also}

$\bar{\sigma}_{N}=\sigma_{N}$

that is, the nominal stress at failure does not depend on the structure size We say in this case that there is no size effect. This is characteristic for all failure analyses according to elasticity with allowable stress (or strength) limit, plasticity, and classical continuum damage mechanics (as well as viscoelasticity and viscoplasticity, because time has no effect on this analysis).

\section{LINEAR-ELASTIC FRACTURE MECHANICS}

In this case, the constitutive relation and the condition of no failure can be written as

$\sigma_{i j}=D_{i j k m} \varepsilon_{k m}$

$J<G_{f}$

in which $D_{i j k m}=$ fourth-order tensor of elastic constants; $G_{f}=$ fracture energy (considered as a material property); and $J=J$-integral

$J=\oint\left(\frac{1}{2} \sigma_{i j} \varepsilon_{i j} d y-\sigma_{i j} n_{j} \partial_{1} u_{i} d s\right)$

[e.g., Kanninen and Popelar (1985); Knott (1973)]. Using the transformation rules in (13)-(15), we find that, for similar contours, $J$ transforms as

$\tilde{J}=\oint\left[\frac{1}{2}\left(\lambda^{m} \sigma_{i j}\right)\left(\lambda^{m} \varepsilon_{i j}\right) \lambda d y-\lambda^{m} \sigma_{i j} n_{j} \lambda^{-1} \partial_{1}\left(\lambda^{m+1} u_{i}\right) \lambda d s\right]$ 


$$
=\Lambda^{2 m+1} \oint\left(\frac{1}{2} \sigma_{i j} \varepsilon_{i j} d y-\sigma_{i j} n_{j} \partial_{1} u_{i} d s\right)=J \lambda^{2 m+1}
$$

Since both $J$ and $J$ must satisfy the same inequality, that is, $j<G_{f}$ and $J$ $<G_{f}$ in all cases, it is obviously necessary and sufficient that $2 m+1=0$, that is

$m=-1 / 2$

Thus, according to (14) and (15), the transformation laws for linear-elastic fracture mechanics are

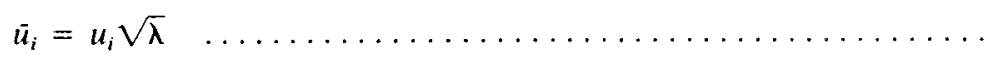

$$
\begin{aligned}
& \bar{\varepsilon}_{i j}=\varepsilon_{i j} / \sqrt{\lambda} \\
& \overline{\boldsymbol{\sigma}}_{i j}=\sigma_{i j} / \sqrt{\lambda} \\
& \bar{p}_{i}=p_{i} / \sqrt{\lambda} \\
& \bar{f}_{i}=f_{i} \lambda^{-3 / 2} \\
& \bar{U}_{i}=U_{i} \sqrt{\lambda} \\
& \bar{\sigma}_{N}=\frac{\sigma_{N}}{\sqrt{\lambda}}
\end{aligned}
$$

where $\lambda=\bar{D} / D$. So the nominal stress at failure depends on the structure size $D, \sigma_{N} \sim 1 / \sqrt{D}$ or

$\log \sigma_{N}=$ constant $-\frac{1}{2} \log D$

In the plot of $\log \sigma_{N}$ versus $\log D$, the linear-elastic fracture mechanics failures are represented by a straight line of slope $-1 / 2$, while all stress- or strain-based failure criteria correspond to a horizontal line (Fig. 2).

The foregoing argument can be generalized to nonlinear elastic behavior, to which the $J$-integral is also applicable. For the case of LEFM, the same result can alternatively be obtained in a more elementary manner. The energy release rate can be calculated by imagining a small crack advance of length $h$ to happen in the following manner: (1) A slit of length $h$ is cut ahead of the crack but is held closed; (2) the normal stresses $\omega_{y}$, acting across

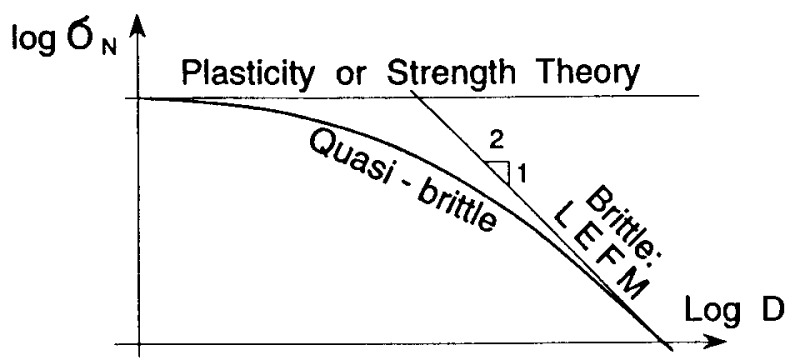

FIG. 2. Size-Effect Laws (Asymptotes) for Limiting Cases of: (a) Strength Criterion; (b) LEFM; and (c) Transitional Size-Effect Curve for Nonlinear Fracture Mechanics the slit are then gradually reduced in proportion to $(1-\tau)$, where $\tau$ is a parameter growing from 0 to 1 ; (3) at the same time, because the body is linearly elastic, the opening displacements of the crack faces grow in proportion to $\tau$ until they reach the final opening displacements $u_{y}$ of a crack with the tip advanced by $h$. The work of $\sigma_{y}$ on $u_{y}$ at both crack faces gives the energy release per length $h$, which must be consumed by the fracture process. Because $\int_{0}^{1}(1-\tau) d \tau=1 / 2$ and the stresses $\sigma_{y}$ work on both crack faces, the work per unit crack advance, i.e., the energy release rate, is

$\mathcal{G}=\lim _{h \rightarrow 0} \frac{1}{h} \int_{0}^{h} \sigma_{y} u_{y} d x$

[e.g., eq. (4.5.2) in Knott (1973) or eq. (12.1.7) in Bažant and Cedolin (1991)] where $x=$ coordinate in the crack direction. Using the foregoing transformation rules, we find that for the scaled structure the energy release rate is

$$
\begin{aligned}
& \overline{\mathscr{G}}=\lim _{h \rightarrow 0} \frac{1}{h} \int_{0}^{h}\left(\lambda^{m} \sigma_{y}\right)\left(\lambda^{m+1} u_{y}\right) d x \ldots \\
& \overline{\mathscr{G}}=\lambda^{2 m+1} \lim _{h \rightarrow 0} \frac{1}{h} \int_{0}^{h} \sigma_{y} u_{y} d x=\lambda^{2 m+\mathrm{J}}
\end{aligned}
$$

which must be the same as the energy release rate given by the preceding equation. Consequently, $\lambda^{2 m+1}=1$ or $m=-1 / 2$.

\section{DIMENSIONAL ANALYSIS}

In an alternative way that is shorter but more abstract (and to a novice less convincing, the size effect can be determined by dimensional analysis. When the structure is elastic-plastic, its failure is governed by the yield stress $\tau_{0}$, whose metric dimension is $N / \mathrm{m}^{2}$. The failure also depends on the nominal stress $\sigma_{N}$, whose metric dimension is also $\mathrm{N} / \mathrm{m}^{2}$. Further, it depends on the characteristic structure-size dimension $D$ and other dimensions such as span $L$, notch length $a$, as well as various other geometric characteristics, all of which have the metric dimension of $m$.

The number of nondimensional variables governing the problem can be determined from Buckingham's II theorem of dimensional analysis [Buckingham (1914, 1915), see also Bridgman (1992); Porter (1933); Giles (1962); Streeter and Wylie (1975); Barenblatt $(1979,1987)$; Iyanaga and Kawada (1980)]. This theorem states that the number of nondimensional variables governing any physical problem is equal to the total number of variables (in these cases five) minus the number of parameters with independent dimensions (in these cases two). Thus, it turns out that the failure condition must have the form

$\Phi\left(\frac{\sigma_{N}}{\tau_{0}}, \frac{L}{D}, \frac{a}{D}, \ldots\right)=0$

where $\Phi=$ function. Since $\tau_{0}$ is a constant, and for geometrically similar structures also $L / D, a / D, \ldots$ are constants, it follows that the nomina stress at failure $\sigma_{N}$, must be proportional to $\tau_{01}$, and therefore a constant when the structure size $D$ is varied. 
In linear-elastic fracture mechanics, the failure is determined by the value of the critical stress intensity factor $K_{t f}$, the metric dimension of which is $\mathrm{Nm}^{-3 / 2}$. The other quantities determining failure are the same as before, including $\sigma_{N}, D, L, a$, and so forth. Again, the number of nondimensional variables on which the failure can depend follows from Buckingham's II theorem and it turns out that the failure condition must now have the form

$\Phi\left(\frac{\sigma_{N} \sqrt{D}}{K_{i_{f}}}, \frac{L}{D}, \frac{a}{D}, \ldots\right)=0$

Since $K_{\text {If }}$ is a material constant, and since the ratios $L / D, a / D, \ldots$ are all constant for geometrically similar structures, it follows that $\sigma_{N} \sqrt{D}$ must also be constant. Hence, $\sigma_{N} \sim D^{-1 / 2}$, which agrees with what we have already shown (Bažant 1983, 1984; Carpinteri 1984, 1986).

The dimensional analysis, however, is too general for revealing certain special features that determine the choice of variables. We will see an example of that when we analyze floating plates.

\section{NONLINEAR FRACTURE MECHANICS}

In nonlinear fracture mechanics, the criterion of crack propagation is characterized by both an energy quantity (the fracture energy $G_{f}$ ) and a stress quantity (strength $f_{i}^{\prime}$ or yield stress $f_{y}$ ). At first one might think that the size effect would be a power law with a constant exponent intermediate between 0 and $-1 / 2$. However, this is not true. Because the ratio $G_{f} / f^{\prime}$, has the dimension of length (in the metric system, it is $N / m$ divided by $\mathrm{N} / \mathrm{m}^{2}$ ), a characteristic length is present in the problem, and so the assumptions underlying (7) are invalid. Hence, the scaling law cannot be a power law.

Previous studies (Bažant 1983, 1984; Bažant 1987; Bažant and Pfeiffer 1987; Bažant and Kazemi 1990) have shown that the scaling law represents a gradual transition from the strength theory to LEFM. This transition has the shape of the curve plotted in Fig. 2, which was experimentally obtained for notched three-point-bend specimens already by Walsh (1979). This curve approaches asymptotically the horizontal line for the strength theory when the size is becoming very small, and the inclined straight line for LEFM when the size is becoming very large. A general exact expression for this curve cannot be obtained, however, under certain simplifying assumptions the following approximate size-effect law (Bažant 1983, 1984) can be derived:

$$
\sigma_{N}=C_{0}(1+\beta)^{-1 / 2} \quad \beta=\frac{D}{D_{0}}
$$

where $\beta=$ relative size; and $C_{0}, D_{0}=$ positive constants. This simple sizeeffect law, whose applicability range is surprisingly broad (but not unlimited), has been extensively verified and applied for quasibrittle materials such as concrete, rocks, ice, tough ceramics, and composites, in which the fracture-process zone has a nonnegligible size and consists of distributed microcracking. This law has been shown to describe well the typical brittle failures of concrete structures, particularly the diagonal shear failure of beams, torsional failure of beams, punching shear failure of slabs, pullout of bars and anchors, failure of bar splices, certain types of compression failures, failure of short and slender columns, and beam and ring failures of pipes. It has also been shown that this law can be exploited for unambiguous definition of material-fracture characteristics, especially the fracture energy (or fracture toughness) and the effective length of the fracture process zone, and for their determination from the peak loads measured on similar specimens of different sizes (Bažant and Kazemi 1990).

The general derivation of the size-effect law for quasibrittle structures yields the asymptotic expansion $\sigma_{N}=C_{0}\left(\beta+1+b_{1} \beta^{-1}+b_{2} \beta^{-2}+b_{3} \beta^{-3}\right.$ $+\ldots)^{-1 / 2}$, where $b_{1}, b_{2}, \ldots$ are constants. This expansion can describe the curve in Fig. 2 for a very broad size range (Bažant 1987). Truncating the foregoing expansion after the second term, one gets formula (32), which appears to suffice, in the case of concrete or rock, for size ranges up to about 1:20. Another empirical generalization yields $\sigma_{N}=C_{0}\left(1+\beta^{r}\right)^{-1 / 2 r}$ (with $r=$ constant), which is more efficient for describing behavior over a very broad size range. This formula reduces to (32) when $r=1$, which was found to be approximately the optimum value for the fitting of the available test data for concrete (Bažant and Pfeiffer 1987).

\section{STRUCTUAES WITH CRITICAL CRACK SIZE INDEPENDENT OF}

\section{STRUCTURE SIZE AND WEIBULL THEORY}

There is a fundamental difference between the classical applications of fracture mechanics to metallic structures and the modern applications to quasibrittle structures, such as concrete structures:

- In the former, the maximum load occurs (or failure must be assumed to occur) while the crack size is still negligible compared to the structural dimensions (Fig. 3), and is determined by material characteristics such as the spacing of major defects, the grain size, or the ratio of fracture energy to yield stress.

- In the latter, there is large stable crack growth (with distributed damage) before the maximum load is reached, and the maximum load occurs when the crack extends over a significant portion of the cross section (in concrete structures it is typically $50-90 \%$ )

Consider now geometrically similar metallic structures of different sizes, made of the same material. The cracks at maximum load are, in each of them, roughly of the same size, and they are so small that the disturbance of the stress field caused by the crack is negligible and the energy release

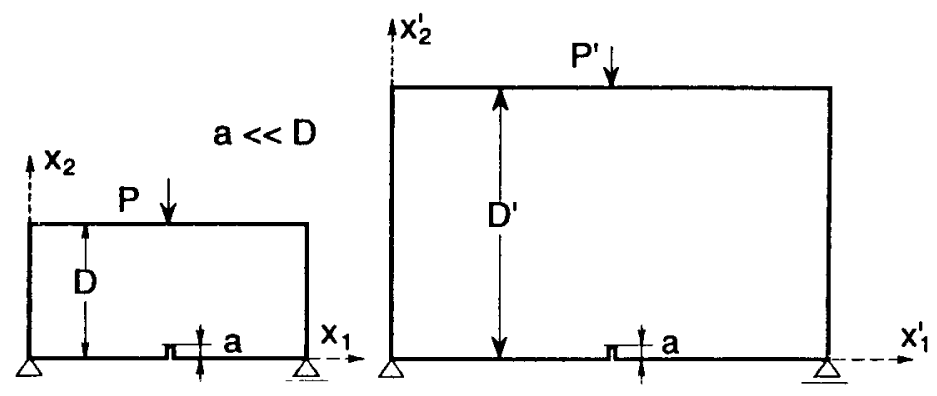

FIG. 3. Geometrically Similar Structures with Very Small Cracks Whose Size Is a Material Property 
caused by the crack is much smaller than the strain energy stored in the structure. In that case, the energy release rate ' can be approximately determined from the stress $\sigma$ (maximum principal stress) that is calculated for the crack location as if no crack existed. Then, considering for example a crack of length $2 a$ in two dimensions, the stress-intensity factor (obtained from the energy release as $K_{I}=\sqrt{E^{(}(G)}$ is approximately calculated from the formula $K_{I}=\sigma \sqrt{\pi a}$, which is exact for a crack in a homogeneously stressed infinite solid. The condition of no failure is written as $K_{t}<K$ where $K_{c}=$ given fracture toughness of the material. Obviously, this condition of no failure is equivalent to

$\sigma<f_{u} \quad$ with $\quad f_{u}=K_{r}(\pi a)^{-1 / 2}$

This is the same as the strength criterion, with $f_{u}$ regarded as the strength of the material.

In some other situations, the crack size at maximum load is not negligible, but is independent of the structure size. Then again, the fracture-mechanic failure criterion is equivalent to the strength criterion, which means that the scaling law is such that there is no size effect on the nominal strength.

In the situations just discussed, in which the critical crack size is independent of the structure size, there can be size effect on the nominal strength but it is not deterministic. Rather, it is caused by randomness of material strength, as described by Weibull-type statistical theories (Weibull 1939; Freudenthal 1968; Bolotin 1969; Elishakoff 1983).

The Weibull law for the spatial density of material-failure probability in general involves a stress threshold below which the failure probability is zero. In practical applications, this threshold is almost always taken as zero, because the test data can be matched by this law also almost equally well with very different threshold values. It is interesting to note that, for a zero threshold, the size effect predicted by the Weibull theory is a power law [e.g., Bažant, Xi and Reid (1991)]. It follows that, according to (7), the Weibull theory for a zero threshold implies that no characteristic structure dimension exists. But this implies that the Weibull theory cannot apply to structures in which the fracture-process zone size has a certain nonnegligible characteristic dimension. Indeed, the statistical size effect is significant only when the structure fails while the crack is still very small, such that the stress redistribution caused by the crack is globally insignificant and the energy release caused by the crack is negligible compared to the total energy in the structure.

Randomness of the material strength is, of course, an inevitable property of materials and its influence is never exactly zero. In quasibrittle structures, however, the Weibull-type statistical size effect is overshadowed by the size effect due to energy release and gets completely suppressed as the size approaches infinity. Proposing a nonlocal adaptation of Weibull theory in which the material failure probability depends on the strain average over a certain characteristic neighborhood of the point rather than on the local stress, Bažant and Xi (1991) derived the following approximate formula:

$\sigma_{N}=C_{0}\left(\beta^{2 n / m}+\beta\right)^{-1 / 2} \quad \beta=\frac{D}{D_{0}}$

in which $C_{0}, D_{0}, m$, and $n=$ positive constants; $n=$ number of dimensions $(1,2$, or 3$)$; and $m=$ Weibull modulus of the material. Normally, the exponent $2 n / m$ is much less than 1 . According to this formula, the classical Weibull-type statistical size-effect $\sigma_{N} \propto D^{-n} m$ is approached asymptotically for sufficiently small structures $(\beta \rightarrow 0)$. But the available test results for concrete structures show this asymptotic behavior to apply, in theory, only to structure sizes that are less than the smallest practical size. In other words, the material strength is random but causes no significant size effect, for any size range. For large structures $(\beta \rightarrow \infty)$, the last equation indicates that $\sigma_{N}$ $\propto D^{-1 / 2}$, that is, the statistical size effect asymptotically disappears. The reason, briefly, is that a significant contribution to the Weibull-type probability integral comes only from the fracture-process zone, which is large but, for structures of different sizes, has roughly the same size.

\section{SOME ANOMALOUS SIZE EFFECTS OF POWER TYPE}

\section{2-D Fracture Problems with Length Parameter in Third Dimension}

Floating Plate under Concentrated Load

Fracture of an infinite floating clastic plate, for which the only geometric dimension $D$ is the plate thickness $h$, is a two-dimensional problem for which $\sigma_{N}$ is not proportional to $D^{-1 / 2}$. This is contrary to our previous general conclusion for two-dimensional fracture problems. The reason is that the plate thickness is merely a parameter determining the bending stiffness, but not a dimension in the two-dimensional domain of coordinates $x$ and $y$ in which the problem is defined mathematically. This property is important for the mechanics of sea ice in the arctic.

Since the buoyancy force depends on the deflection linearly, a plate floating on water of specific weight $\rho$ is equivalent to a plate on a Winkler elastic foundation of stiffness $\rho$. The properties of the plate are characterized by the cylindrical bending stiffness $D=E h^{3} / 12\left(1-v^{2}\right)$ and the Poisson ratio v where $E=$ Young's elastic modulus. In terms of these parameters one can define the quantity (Hetényi 1946)

$L=\left(\frac{4 D}{\rho}\right)^{1 / 4}=\left(\frac{E h^{3}}{3 \rho\left(1-v^{2}\right)}\right)^{1 / 4}$

which has the dimension of length. It may be called the decay length because it characterizes the distance over which the amplitude of a local disturbance to the plate decays by the ratio $1 / e$.

The deflection surface $w(x, y)$ of a floating plate under distributed load $p(x, y)$ is governed by the differential equation

$D \nabla^{4} w+\rho w=p(x, y)$

For a vertical concentrated load $P$ at point $x=y=0$, we have $p(x, y)=$ $P \delta(x, y)$ where $\delta(x, y)=$ two-dimensional Dirac delta function, such that $\iint \delta(x, y) d x d y=1$. As known from experiments, radial cracks forming a certain central angle $\phi_{n}$ grow in a stable manner as the load is increased. The maximum load occurs when circumferential cracks at a certain radial distance $a=a_{c}$ begin to grow. As these cracks grow further, the load decreases at increasing load-point displacement.

Introducing nondimensional variables $\xi=x / L, \eta=y / L$ and $\zeta=w / L$, and noting that $p=\delta(x, y) P / L^{2},(36)$ is transformed to

$\bar{\nabla}^{4} \zeta+4 \zeta=\bar{\delta}(\xi, \eta)\left(\frac{P L}{D}\right)$

where $\bar{\delta}(\xi, \eta)=L^{2} \delta(L \xi, L \eta)$ and $\bar{\nabla}^{4}=L^{4} \nabla^{4}=$ biharmonic operator on 
coordinates $\xi$ and $\eta$. Let now $\zeta=F(\xi, \eta ; \alpha)$ represent the solution of the differential equation

$\bar{\nabla}^{4} \zeta+4 \zeta=\bar{\delta}(\xi, \eta)$

for a plate containing a crack of relative length $\alpha=a / L$ (the proper freeboundary conditions must also be written for the crack surfaces). Consider now that the boundary condition at infinity is $w=0$. Then the solution of differential equation $(37)$ is $\zeta=F(\xi, \eta ; \alpha)(P L / D)$.

Now the complementary energy of the floating plate may be calculated as II $=P w_{0} / 2=P L \zeta_{0} / 2=F_{0}(\alpha) P^{2} L^{2} /(2 D)$ where we denoted $F_{0}(\alpha)=$ $F(0,0 ; \alpha)$. For the energy release rate we now have the condition [oll/ $\partial a]_{p},=[\partial I / / \partial \alpha]_{p} / L \stackrel{=}{=} F_{0}^{\prime}(\alpha) P^{2} L /(2 D)=h G_{r}$. Solving $P$ from this equation, calculating the nominal strength $\sigma_{N}=P / h^{2}$ and expressing $L$ and $D$ in terms of $h$, we finally get the following result (Bažant 1992a):

$\sigma_{N}=C_{0} h^{-3 / 8} \quad$ with $\quad C_{0}=\left(\frac{\rho}{108}\right)^{1 / 8}\left(\frac{E}{1-v^{2}}\right)^{3 / 8} \sqrt{\frac{G_{f}}{F_{0}^{\prime}(\alpha)}}$

Thus, if the ratio $\alpha$ of the radial crack length $a$ at maximum load to the decay length $L$ is the same for all ice thicknesses $h$ (i.e., the cracks that occur for different thicknesses at maximum load are geometrically similar), the size effect on nominal strength is of the type $h^{-3 / 8}$, and not of the type $h^{-1 / 2}$. This is so in spite of the fact that the only geometric dimension present in the problem is $h$. But in terms of the decay length $L$ we have

$\sigma_{N} \propto L^{-1 / 2}$

because $h \propto L^{4 / 3}$, according to $(35)$. So in terms of $L$, which represents the only length parameter in the plane $(x, y)$, the size effect agrees with the general property we deduced before.

It must be emphasized that if the ratio $\alpha$ for different $h$ is not the same, then the size effect is, of course, different.

\section{Thermal Bending Fracture of Floating Plate}

A size effect of the type $h^{-3 / 8}$ is encountered not only for fracture caused by vertical loading, but also for fracture caused by thermal bending moments $M_{T}$ due to temperature gradient across the plate thickness. Before fracture, the floating plate is undeflected $(w=0)$. Thermal fracture relaxes the thermal bending moments in the vicinity of the fracture and causes release of the strain energy due to the thermal bending moments.

The governing differential equation for the two-dimensional deflection surface $w(x, y)$ is $D \nabla^{4} w+\rho w=0$. The plate is infinite, with the boundary condition $w=0$ at infinity. At the crack faces $\Gamma_{0}$, which can in general be curved, the boundary conditions are $D w_{, n}=M_{r}$ and $w_{, m m}+(1-v) w_{n t}$ $=0$, where subscripts following a comma denote partial derivatives, and $n$ and $t$ are the coordinate axes normal and tangential to the crack face.

Introduce now dimensionless variables $\xi=x / L, \eta=y / L$ and $\zeta=w / L$, and note the transformation of derivatives: $\partial / \partial x=L-1 \partial / \partial \xi$, and so forth. Transforming the boundary value problem to these variables, we obtain the governing partial differential equation

$\bar{\nabla}^{4} \zeta+4 \zeta=0$

with boundary conditions $\zeta=0$ at infinity. At crack faces $\Gamma_{0}$, the boundary conditions in dimensionless coordinates are $\zeta_{. v v}=\frac{L}{D} M_{r}$

and

$\zeta_{. v w v}+(1-v) \zeta_{. v \tau \tau}=0$

where $v$ and $\tau=$ coordinate axes normal and tangential to the crack faces $\Gamma_{0}$ in the dimensionless space $(\xi, \eta)$.

Due to linearity of (41) and (42), the solution $\zeta$ is proportional to $M_{\text {r }}$ Therefore, it is convenient to define: $\zeta=F(\xi, \eta ; \bar{a})=$ solution of differential equation (41) for the relative crack length $\bar{a}=a / L$ and for the aforementioned boundary conditions, except that the first boundary condition in (42) is replaced by $\zeta_{, v v}=1$. In such a boundary value problem, there are no physical constants, and so the solution $F$ is independent of the size and material properties, and depends only on $\bar{a}$. Then the solution for the actual boundary conditions (42) is

$\zeta=\frac{L}{D} M_{T} F(\xi, \eta, \bar{a})$

Now, by transformations of coordinates, we get for the crack faces: $\Gamma, \zeta$, $=F_{, v} M_{T} L / D, \zeta_{, n}=\zeta_{. v} / L ;$ and $\theta=w_{. n}=L \zeta_{. n}=F_{, v} M_{T} L / D=$ rotation at the crack face about the tangential axis $\tau$. The total complementary energy release due to fracture, $1 \mathrm{l}$, is equal to the work of the released thermal bending moment, as it is reduced to zero, on rotation $\vartheta$, i.e., $I I=\int_{a}$ $1 / 2 M_{T} \vartheta d a$. From this we get the energy release per unit length of fracture: $\partial \Pi / \partial a=1 / 2 M_{T} \vartheta=G_{f} h$. Substituting now the foregoing expression for $\vartheta$ solving the resulting equation for $M_{T}$, and expressing $M_{T}$ in terms of $\Delta T_{c \mathrm{c}}$, and $L$ and $D$ in terms of $h$, we obtain the following result (Bažant 1992b):

$\Delta T_{c r}=\frac{2^{1 / 4}(1-\nu)^{3 / 8}}{\sqrt{F_{, n}(\xi, \eta ; \bar{a})}} C_{1} h^{-3 / 8}$

in which $C_{1}$ is a size-independent constant

$C_{1}=\frac{(1-v)^{5 / 8} \rho^{1 / 8} \sqrt{G_{f}}}{\sqrt{2}\left[3\left(1-v^{2}\right)\right]^{3 / 8} E^{5 / 8} \alpha I_{T}}$

This proves in general that thermal bending fracture of floating ice plate exhibits a $(-3 / 8)$ power size effect, provided that either $F$ is independent of the crack length (which occurs for a semiinfinite crack in an infinite plate) or the crack length $a$ is proportional to the decay length $L$ (rather than to thickness $h$ ) (Bažant 1992a, b). In terms of the decay length $L$, the size effect is of the type $\Delta T_{c r} \propto L^{-1 / 2}$, similarly as before. This simple conclusion is not surprising since, due to Kirchhoff's assumption, the plate problem is two-dimensional and, in the plane $(x, y)$ of the boundary value problem, length $L$ is the only characteristic length present ( $h$ enters only indirectly, through $D$ ).

If we carried out the dimensional analysis according to the Buckingham's II theorem in the usual manner, taking the only geometric dimension present as the characteristic dimension of the structure, we would have found for $\sigma_{N}$ a result different from $h^{-3 / 8}$. However, we could as well have taken the decay length $L$ as the characteristic dimension, and then we would have obtained the correct size effect $h^{-3 / 8}$. So the result would have been incon- 
clusive. It is necessary to know something about the mechanics of the problem to realize that $L$ rather $h$ must be taken as the characteristic dimension, and only then the correct size effect $h^{-3 / 8}$ is obtained from Buckingham's II theorem.

\section{Zone of Splitting Compression Cracks}

An anomalous size effect of power type has also resulted from an analysis of boreholc breakout in rock under certain simplifying hypotheses. In an infinite elastic space that is initially under uniform triaxial stress with minimum principal stress $\sigma_{N}$ a cylindrical borehole of diameter $D$ is drilled. This causes a zone of parallel splitting cracks to form on the sides of the borehole. For various borehole diameters $D$, these zones are considered to be similar. The growth of the cracking zone causes a release of the stored energy which must be equal to the energy consumed by the growth of the cracks. Using this condition and assuming the splitting cracks to follow LEFM, Bažant et al. (1993) showed that

$\sigma_{N} \propto D^{-2 / 5}$

The reason that the exponent is not $-1 / 2$ is twofold: (1) There is not one but many cracks; and (2) the spacing $s$ of the cracks is not proportional to $D$ but to $D^{4 / 5}$, which is deduced from the analysis of buckling of the intact rock slabs between the parallel cracks.

\section{CONCLUSIONS}

1. The scaling law is a power law if, and only if, a characteristic dimension is absent. This applies, for example, to elasticity with a strength limit, plasticity, continuum damage mechanics (without nonlocal concepts), and linear-elastic fracture mechanics.

2. In all the theories in which the failure condition is expressed in terms of stress or strain only, the nominal strength $\sigma_{N}$ of the structure is independent of its size, i.e., there is no size effect. This includes elasticity with a strength limit (or allowable stress limit), plastic limit analysis, and continuum damage mechanics in the classical form (without nonlocal concepts).

3. In linear-elastic fracture mechanics, in which the failure critcrion is expressed in terms of energy per unit area (fracture energy), the scaling law is such that $\sigma_{N} \propto(\text { size })^{-1 / 2}$, provided that the cracks in structures of different sizes are geometrically similar. However, there is no (deterministic) size effect in the classical fracture mechanics applications to metallic structures, in which the critical crack size is very small compared to the structure size and is a material property, independent of the structure size.

4. When the failure condition involves both the stress (or strain) and the energy per unit area, the scaling law represents a gradual transition between the strength theory, which is asymptotically approached for very small sizes, and LEFM, which is asymptotically approached for very large sizes.

5. Floating elastic plates with large bending fractures and a negligible process-zone size exhibit a scaling law of the type $\sigma_{N} \propto(\text { size })^{-3 / 4}$. This anomaly is caused by the fact that the plate thickness, which is the only geometric dimension present, is not a dimension in the two-dimensional domain of the boundary-value problem. Another anomaly, in which the scaling law is $\sigma^{N} \propto(\text { size })^{-2 / 5}$, arises in the problem of breakout of boreholes in rock if it is assumed that the failure is due to a zone of parallel splittingcompression cracks whose spacing is governed by buckling considerations, and that the zones are similar for different sizes.

\section{ACKNOWLEDGMENT}

Financial support under ONR Grant N00014-91-J-1109 to Northwestern University is gratefully acknowledged. Furthermore, the general study of power law was partly carried out during the writer's visiting appointment at Lehrstuhl A für Mechanik. Technische Universität München, supported under Humboldt Award of senior U.S. scicntist, and some applications were partially supported by NSF S\&T Center for Advanced Cement-Based Materials at Northwestern University.

\section{APPENDIX I. REFERENCES}

Barenblatt, G. I. (1979). Similarity, self-similarity, and intermediate asymptotics Consultants Bureau, New York, N.Y.

Barenblatt, G. I. (1987). Dimensional analysis. Gordon and Breach, New York, N. Y.

Bažant, Z. P. (1983), "Fracture in concrete and reinforced concrete." IUTAM Prager Symp. on Mechanics of Geomaterials: Rocks, Concretes, Soils. Z. P. Bažant, ed., Northwestern Univ., Evanston, Ill., 281-316 (Eq. 16).

Bažant, Z. P. (1984). "Size effect in blunt fracture: concrete, rock, metal." J. of Engrg. Mech., ASCE, 110, 518-535.

Bažant, Z. P. (1992a). "Large-scale fracture of sea ice plates." Proc., IIth IAHR Int. Ice Symp., T. M. Hrudey, ed., Univ. of Alberta, Edmonton, Alberta, Canada,

Bažant, Z. P. (1992b). "Large-scale thermal bending fracture of sea ice plates." $J$ of Geophysical Res., 97(C11), 17,739-17,751.

Bažant, Z. P. (1987). "Fracture energy of heterogeneous matcrial and similitude." SEM-RILEM Int. Conf. on Fracture of Concrete and Rock, S. P. Shah and S. E. Swartz, ed., Soc. for Exper. Mech., Bethel, Ct., 390-4012.

Bažant, Z. P., and Pfeiffer, P. A. (1987). "Determination of fracture energy from size effect and brittleness number." ACI Mat. Jour. 84, 463-480.

Bažant, Z P., and Kazemi, M. T. (1990). "Size Jour., 84,463 Bažant, Z. P., and Kazemi, M. T. (1990). "Size effect in fracture of ceramics and its use to determine fracture energy and effective process zone length." $J$. of $\mathrm{Am}$.

Bažant, Z. P., Lin, F.-B., and Lippmann, H. (1993). "Fracture energy release and size effect in borehole breakout." Int. J. of Num. and Anal. Methods in Geomechanics, 17, 1-14.

Bažant, Z. P., Xi, Y., and Reid, S. G. (1991). "Statistical size effect in quasi-brittle structures: I. Is Weibull theory applicable?" J. Engrg. Mech., ASCE, 117(11), $2609-2622$.

Bažant, Z. P., and Xi, Y. (1991). "Statistical size effect in quasi-brittle structures: II. Nonlocal theory." J. Engrg. Mech., ASCE, 117(11), 2623-2640.

Bridgman, P. W. (1922). Dimensional analysis. Yale Univ. Press, New Haven, Conn.

Bolotin, V. V. (1969). Statistical methods in structural mechanics. Holden Day, San Francisco, Calif.

Buckingham, E. (1914a). "On physically similar systems; illustrations of the use of empirical equations." Physical Rev., Ser. 2, IV(4), 345-376.

Buckingham, E. (1915b). "Model experiments and the form of empirical equations." Trans., ASME 37, 263-296.

Carpinteri, A. (1984). "Scale effects in fracture of plain and reinforced concretc structures." Fracture mechanics of concrete: structural application and numerical calculation, G. C. Sih and A. DiTommaso, eds., Martinus Nijhoff Publ., The Hague, The Netherlands, 95-140. 
Carpinteri, A. (1986). Mechanical damage and crack growth in concrete, Martinus Nijhoff Publ., Boston, Mass. (chapter 8 )

Elishakoff, I. (1983). Probabilistic methods in the theory of structures. John Wiley and Sons, New York, N.Y

Encyclopedic dictionary of mathematics. (1980). S. Lyanaga and Y. Kawada, eds. MIT Press, Cambridge, Mass., Sec. 120, 412-413.

Freudenthal, A. M. (1968). "Statistical approach to brittle fracture." Fracture - An advanced treatise. H. Liebowitz, ed., 2, Academic Press, San Dicgo, Calif., 591 619.

Giles, R. V. (1962). Theory and problems of fluid mechanics and hydraulics. McGrawHill, New York, N.Y. (chapter 5)

Hetényi, M. (1946). Beams on elastic foundation. Univ. of Michigan Press, Ann Arbor, Mich.

Kanninen, M. F. and Popelar, Carl H. (1985). Advanced fracture mechanics. Oxford Univ. Press, New York, N.Y.

Knott, J. F. (1973). Fundamentals of fracture mechanics. Butterworth, London. England.

Porter, A.

England. New York. N.Y. (chapter 4).

Walsh, P. F. (1979). "Fracture of plain concrete." The Indian Concrete J., 46(11), 469,470 , and 476 .

Weibull, W. (1939). "A statistical theory of the strength of materials." Royal Swedish Academy of Engrg. Sci., Proc., Stockholm, Sweden, 151, 1-45. 